\section{P51 REDUCED EFFECTIVENESS OF THE PRIMARY-CARE REGISTRY FOR TARGETED LTBI SCREENING OF HIGH RISK IMMIGRANTS WITH HIV CO-INFECTION}

doi:10.1136/thoraxjnl-2012-202678.192

RK Panchal, G Woltmann, P Haldar. Institute for Lung Health, Glenfield Hospital, Leicester, United Kingdom

Introduction The impact of screening for latent tuberculosis infection (LTBI) in immigrants for tuberculosis (TB) prevention in the UK is dependent on effective strategies for identifying at-risk groups. Here we investigate effectiveness of the primary-care (PC) registry to identify immigrants for enrolment to LTBI screening at the time of GP registration, based on their HIV status.

Methods We performed a 11-year retrospective cohort study of PC registrations, cross-referenced with foreign-born TB (FB-TB) notifications for immigrants entering the UK after 1999 that had HIV testing performed. We assumed LTBI screening to prevent prospective TB would be effective if notifications occurred $\geq 6$ months after $\mathrm{PC}$ registration. The primary outcome was the proportion of FB-TB preventable with screening at PC registration and compared between subgroups stratified according to HIV status.

Results 624 of 857 FB-TB cases were HIV tested (72.8\%) and 84 were HIV seropositive (13.4\%). 56 (67\%) HIV positive FB-TB cases occurred in immigrants from TB incidence regions 500/100,000. Overall 511 (63\%) FB-TB cases were preventable if screened at PC registration. The proportion with unpreventable TB was significantly higher for the HIV positive compared to the HIV negative FB-TB subgroup; [ $19 \%$ vs $10 \%$; RR $(95 \% \mathrm{CI})=1.89$ (1.25 to 2.84$)$, $\mathrm{p}=0.003]$. Compared with patients that were HIV negative, time to PC registration after UK entry was longer [median (IOR); 1515 (555-2202) days vs 415 (36-1558) days; $\mathrm{p}<0.005)$ and time to disease notification shorter [median (IQR); 587 (208-1182) days vs 1163 (669-1854) days, $p<0.005]$ for HIV positive FB-TB patients.

Conclusion Targeted LTBI screening at the time of primary-care registration may be a less effective preventative strategy for HIV positive immigrants at highest TB risk. Our data supports the need to promote early registration and extended screening to include HIV and other blood-borne viruses as part of an integrated immigrant screening programme.

\section{P52 THE USE OF MAGNETIC RESONANCE STUDIES [MR] IN ASSESSING RESPONSE AND GUIDING DURATION OF TREATMENT IN SPINAL TUBERCULOSIS}

doi:10.1136/thoraxinl-2012-202678.193

KA Chengappa, J Lucas, HJ Milburn. Guy's and St. Thomas'NHS Foundation Trust, London, UK

Background Significant delays still occur in the diagnosis of spinal tuberculosis [sTB] and there is little consensus on the follow-up and duration of treatment. Current UK and international guidelines for sTB still recommend standard short course chemotherapy but this does not reflect clinical practise.

Aims and methods We performed a retrospective study at a UK teaching hospital to identify the demographic and clinical features of our sTB population and the value of spinal magnetic resonance studies $[\mathrm{MR}]$ in guiding response to and duration of treatment.

Results Data was available for 26 patients [2005-2011] with an average age of 40 years [60\% male]. Mean time to diagnosis was 9.3 months. At presentation the commonest symptoms were back pain $-26 / 26$, constitutional symptoms [fevers, sweats, weight loss] - 12/26 and neurological symptoms - 12/26. In 13/26 [50\%], the diagnosis was made following an emergency admission. Positive microbiology was obtained in 19/26 and $84 \%$ of these were fully sensitive organisms. $42 \%$ had disease at multiple spinal levels and $73 \%$ had associated paraspinal abscesses. At 6 months only $2 / 26$ [7\%] had MR evidence of resolution of active disease and 11/26 [42\%] had persisting symptoms [back pain and neurological symptoms]. The median number of follow-up scans/patient was 2 and mean duration of treatment was 10.7 months. Of the 24 patients who received $>6$ months treatment, 5 [20\%] still had symptoms after completion [back pain].

Conclusions MR provides a useful means of assessing response to treatment and can help in deciding duration of treatment. However the frequency/intervals at which scans should be done is not clear.

\section{P53 FAST TRACK TB RADIOLOGY: A BIT OF A BONSAI OR A TREE IN BUD?}

doi:10.1136/thoraxjnl-2012-202678.194

GS Bhat, F Oyesanya, J Cleverley, C Cash, J Goldring, I Cropley, MCI Lipman Royal. Free Hospital, London, London

Background Prompt diagnosis is central to effective tuberculosis(TB) management. To minimise clinician related delay, we developed a pathway where imaging suggestive of active TB is flagged for review by specialist radiologists and the TB service at a weekly meeting. If required, the patient is then fast-tracked for clinical assessment by the TB team.

Methods Prospective study of patients referred to our radiology service June 2011-February 2012 with imaging flagged as suspicious of TB. Case management, diagnosis and final outcome were recorded Results 68 subjects, median age 45 years (range 1-91), were studied. Of the flagged images, 38 were chest radiographs, 28 CT chest +/- abdomen, 1 CT head and 1 MRI spine. 32 (47\%) were reported to be highly suspicious for TB. The remaining 36 (53\%) had changes such as apical scarring, pleural effusion and/or mediastinal lymphadenopathy where TB was part of the differential diagnosis. 19 $(28 \%)$ of the radiological referrals were from Primary Care \& 12 (18\%) Accident \& Emergency (A \& E). 59 of 68 (87\%) were seen in a TB clinic at a median time from date of imaging to appointment of 13 days (range $1-100) .18$ (31\%) had a final diagnosis of active TB. 3 were already on TB treatment, and the remaining 15 started a median 24 days from the time of imaging. Apart from TB, common final diagnoses included bacterial or NTM infection, bronchiectasis and sarcoidosis. 4/19 (21\%) of GP and 3/12 (25\%) A \& E referrals had active TB. Patients not seen in a TB clinic were referred to other clinical services (4 cases - all with significant pathology) or no action taken following specialist review of their imaging (5).

Conclusion The fast track service has a high yield for TB and other significant pathologies. Almost half of the reviews arose following Primary Care or A \& E imaging. Prompt specialist imaging review is associated with rapid and appropriate triage to $\mathrm{TB}$ and other services Further work will compare this approach to standard care pathways.

\section{P54 TESTING FOR BLOOD BORNE VIRUSES IN A TB CLINIC: WHY DISCRIMINATE ON THE BASIS OF WHO GETS TB TREATMENT?}

doi:10.1136/thoraxinl-2012-202678.195

'TP Cusack, 'J Sewell, 'S Chaytor, 'J Johnson, 'A Solamalai, 'P MacKenzie, ${ }^{1}$ A Goodburn, 'S Hopkins, 'I Cropley, 'M Lipman. 'Royal Free London NHS Foundation Trust, London, United Kingdom; '2University College London, London, United Kingdom

Introduction Testing for blood borne viruses (BBV), in particular HIV, is a standard of care for people on active tuberculosis (TB) treatment. Although individuals with latent $\mathrm{TB}$ infection and those with suspected TB disease are drawn from similar populations, with presumably similar risks for BBV, there are little reported data on this. Our TB clinic has a policy of routine testing for HIV, Hepatitis $\mathrm{B}(\mathrm{HBV})$ \& Hepatitis C (HCV) on those with a diagnosis of active and latent TB. Here we review our data.

Methods Information on all adults attending TB services between 01/01/2009 and 19/07/2012 at our centre (apart from those known to be HIV infected at the time of TB service encounter) were extracted from hospital clinical and pathology information systems. 